\title{
Genome-Wide Temporal Expression Profiling in Caenorhabditis elegans Identifies a Core Gene Set Related to Long-Term Memory
}

\author{
Virginie Freytag, ${ }^{1,2 \star}$ Sabine Probst, ${ }^{2,5 *}$ Nils Hadziselimovic, ${ }^{2,5}$ Csaba Boglari, ${ }^{2,5}$ Yannick Hauser, ${ }^{1,2}$ Fabian Peter, ${ }^{1,2,5}$ \\ Bank Gabor Fenyves, ${ }^{2,6}$ Annette Milnik, ${ }^{1,2,3}$ Philippe Demougin, ${ }^{5}$ Vanja Vukojevic, ${ }^{1,2,5}$ \\ Dominique J.-F. de Quervain, ${ }^{1,3,4}$ Andreas Papassotiropoulos, ${ }^{1,2,3,5}$ and Attila Stetak ${ }^{1,2,3}$ \\ ${ }^{1}$ Transfaculty Research Platform Molecular and Cognitive Neurosciences, ${ }^{2}$ Division of Molecular Neuroscience, Department of Psychology, ${ }^{3}$ University \\ Psychiatric Clinics, and ${ }^{4}$ Division of Cognitive Neuroscience, Department of Psychology, University of Basel, 4055 Basel, Switzerland, ${ }^{5}$ Biozentrum, Life \\ Sciences Training Facility, University of Basel, 4056 Basel, Switzerland, and ${ }^{6}$ Department of Medical Chemistry, Semmelweis University, 1094 Budapest, \\ Hungary
}

The identification of genes related to encoding, storage, and retrieval of memories is a major interest in neuroscience. In the current study, we analyzed the temporal gene expression changes in a neuronal mRNA pool during an olfactory long-term associative memory (LTAM) in Caenorhabditis elegans hermaphrodites. Here, we identified a core set of 712 (538 upregulated and 174 downregulated) genes that follows three distinct temporal peaks demonstrating multiple gene regulation waves in LTAM. Compared with the previously published positive LTAM gene set (Lakhina et al., 2015), 50\% of the identified upregulated genes here overlap with the previous dataset, possibly representing stimulusindependent memory-related genes. On the other hand, the remaining genes were not previously identified in positive associative memory and may specifically regulate aversive LTAM. Our results suggest a multistep gene activation process during the formation and retrieval of long-term memory and define general memory-implicated genes as well as conditioning-type-dependent gene sets.

Key words: C. elegans; CREB; expression; genes; long-term memory; microarray

Significance Statement

The identification of genes regulating different steps of memory is of major interest in neuroscience. Identification of common memory genes across different learning paradigms and the temporal activation of the genes are poorly studied. Here, we investigated the temporal aspects of Caenorhabditis elegans gene expression changes using aversive olfactory associative long-term memory (LTAM) and identified three major gene activation waves. Like in previous studies, aversive LTAM is also CREB dependent, and CREB activity is necessary immediately after training. Finally, we define a list of memory paradigm-independent core gene sets as well as conditioning-dependent genes.

\section{Introduction}

The identification of genes involved in encoding, storage, and retrieval of memories is of paramount scientific interest in neu-

Received Aug. 17, 2016; revised April 9, 2017; accepted May 10, 2017.

Author contributions: D.J.-F.d.Q., A.P., and A.S. designed research; S.P., N.H., C.B., Y.H., F.P., B.G.F., P.D., and A.S. performed research; V.F., S.P., A.M., V.V., and A.S. analyzed data; V.F., S.P., D.J.-F.d.Q., A.P., and A.S. wrote the paper.

This work was supported by Swiss National Science Foundation Grants 31003A_156579 (A.S.) and 320030_163434 (A.P.). Strains were provided by the Caenorhabditis Genetics (enter, which is funded by the NIH Office of Research Infrastructure Programs (Grant P40 OD010440).

*V.F. and S.P. contributed equally to this work.

The authors declare no competing financial interests.

Correspondence should be addressed to Attila Stetak, University of Basel, Birmannsgasse 8, 4055 Basel, Switzerland.E-mail:a.stetak@unibas.ch. roscience because of its implications for basic and medical research. So far, several microarray studies were performed to identify genes that contribute to learning and memory, longterm potentiation, and long-term plasticity (Cavallaro et al., 2002; Paratore et al., 2006; Burger et al., 2008; Benoit et al., 2011). For example, expression changes of neuronal genes were analyzed in vertebrates such as rats and in mice to detect genes that are differentially expressed after induction of long-term potentiation (Park et al., 2006; Ryan et al., 2011). Moreover, the memory consolidation process in rats, mice, and rabbits was studied at the transcriptional level after different learning paradigms, such as

DOI:10.1523/JNEUROSCI.3298-16.2017

Copyright $\odot 2017$ the authors $\quad 0270-6474 / 17 / 376661-12 \$ 15.00 / 0$ 
passive avoidance learning, fear conditioning, T-maze learning, Morris water maze training, and classical eye-blink conditioning (for review, see Paratore et al., 2006). Importantly, many early microarray studies in vertebrates were performed using arrays limited to a selected subset of known neuronal genes, thus reducing the consistency of gene expression findings across the different memory assays.

The relatively simple nervous system of Caenorhabditis elegans allows associative learning between a variety of volatile or soluble odorants and food. Several olfactory learning assays of long-term associative memory (LTAM) have been established, such as positive olfactory conditioning, in which repeated presentation of 2-butanone as an odorant in association with food leads to longterm attraction to the odorant (Kauffman et al., 2011). Alternatively, under aversive olfactory conditioning, pairing of diacetyl (DA) as an attractant with starvation results in long-term avoidance to DA (Vukojevic et al., 2012). Previously, tests using different memory assays in C. elegans have already led to the discovery of several genes critical for long-term associative learning (for review, see Ardiel and Rankin, 2010).

Like in mammals, long-term memory requires transcriptional gene activation also in C. elegans (Kauffman et al., 2011; Vukojevic et al., 2012). Recently, transcriptome-wide changes directly after olfactory LTAM training have been investigated in C. elegans (Lakhina et al., 2015). Combining positive olfactory training together with genome-wide expression analysis of wild-type and CREB mutant animals, Lakhina et al. (2015) identified CREBdependent or -independent LTAM-induced genes. However, previous studies in C. elegans have not investigated the temporal aspects of gene activation during LTAM.

Here, we performed a microarray-based time course assay with an enriched neuronal mRNA pool, after aversive olfactory associative conditioning in C. elegans. We identified a core set of 712 (538 upregulated and 174 downregulated) genes that was reproducibly regulated with different temporal kinetics during LTAM. The gene regulation followed three different temporal activation patterns suggesting multiple gene activation waves during consolidation of LTAM. Two of these activation peaks coincided with the memory inhibitory effect of actinomycin D that showed a biphasic inhibition of the aversive LTAM. Fifty percent of the identified upregulated genes were previously identified during positive olfactory LTAM (Lakhina et al., 2015) and might therefore, represent stimulus-independent memoryregulated genes. On the other hand, the remaining $50 \%$ of the genes were not related to positive LTAM and may specifically regulate aversive olfactory LTAM. Furthermore, we showed that aversive LTAM is CREB dependent, that CREB phosphorylation increases after conditioning, and that CREB activity is necessary in the first $2 \mathrm{~h}$ after training. In summary, our results suggest the existence of a multistep gene activation process during LTAM and define general memory-implicated genes as well as conditioningtype-specific gene sets.

\section{Materials and Methods}

Reagents, C. elegans growth conditions, and strains used

All reagents used were from Sigma-Aldrich, unless indicated. Worms were maintained using standard methods and grown at $20^{\circ} \mathrm{C}$ (Brenner, 1974). For all the experiments, a synchronized population of young adult hermaphrodite worms was used. Strains used were the wild-type C. elegans Bristol strain, variety N2, gaIs153[pF25B3.3::FLAG::pab-1;sur-5:: GFP] (Von Stetina et al., 2007), gaIs146[myo-3::FLAG::pab-1;sur-5::GFP] (Roy et al., 2002), syg-1(ky652), ppt-1(gk139), rig-5(utr2), ras-2(ok682), eps-8(by160); Ex[opt-2p::EPS-8A::GFP] (Croce et al., 2004), and rpm-1(ju41), syd-2(ok217) ncx-3(utr4), prkl-1(ok3182), ttx-1(oy26)
oyIs17, crh-1(tz2), crh-1(tz2); utrEx80[hsp16.41p::crh-1::FLAG; sur-5:: dsred; myo-3::rfp].

\section{Conditioning of $\mathrm{C}$. elegans and behavior assays}

Long-term conditioning was performed as described previously (Vukojevic et al., 2012), and three independent biological replicates were tested. Briefly, well fed synchronized young adults were starved in the presence of $2 \mu \mathrm{l}$ of DA for $1 \mathrm{~h}$, and the conditioning was repeated separated by a 30 min recovery phase on seeded plates without DA. In the case of the temporal profiling assay (first experiment), the worms were either collected immediately or transferred on nematode growth medium (NGM) plates with abundant food and collected $0.5,1,2,4,8,16$, and $24 \mathrm{~h}$ after conditioning. As controls, worms were exposed for $1 \mathrm{~h}$ to starvation alone (S) or to DA in the presence of abundant food (A). For the second expression analysis, worms were collected 4 or $8 \mathrm{~h}$ after conditioning, starvation, or DA treatment in the presence of food. Pulse treatment with actinomycin $\mathrm{D}$ was performed by soaking worms for $5 \mathrm{~min}$ in M9 supplemented with DMSO alone or $100 \mu \mathrm{g} / \mathrm{ml}$ actinomycin D in DMSO at different times after DA conditioning. After treatment, worms were washed in M9 and placed on seeded NGM plates for additional time, and chemotaxis toward DA of the treated worms was tested $24 \mathrm{~h}$ after the initial DA conditioning. Chemotaxis was assessed by washing well fed young adult worms of different genotypes with CTX buffer $(25 \mathrm{~mm}$ K-phosphate, pH 6.0, $1 \mathrm{mM} \mathrm{CaCl}_{2}$, and $1 \mathrm{mM} \mathrm{MgSO}_{4}$ ) and tested their attraction to $0.1 \%$ DA on $10 \mathrm{~cm}$ CTX agar plates. For the LTAM behavioral assays, well fed young adult worms were tested for their attraction to $0.1 \%$ DA before conditioning (naive) or exposed to $2 \mu \mathrm{l}$ of DA for $1 \mathrm{~h}$ in the absence of food. Conditioning was repeated separated by a $30 \mathrm{~min}$ recovery phase on seeded plates without DA. After conditioning, worms were tested for their preference to DA immediately $(0 \mathrm{~h})$ or worms were washed in M9 and placed on seeded NGM plates for additional time, and their chemotaxis toward DA was tested $24 \mathrm{~h}$ after the initial DA conditioning. The attraction to diacetyl was visualized either as chemotaxis index (CI; Bargmann et al., 1993) or as memory index ( $\mathrm{MI}=\mathrm{CI}_{\text {naive }}-$ $\mathrm{CI}_{\text {delay }} / \mathrm{CI}_{\text {naive }}$; Stetak et al., 2009).

\section{Real-time $q P C R$}

Total RNA was isolated from synchronized adult worms using the Direct-zol RNA MiniPrep kit (Zymo Research Cooperation) with DNase treatment. A total of $400 \mathrm{ng}$ of RNA was supplemented with $20 \mathrm{ng}$ of Phe spike RNA, and the mix was reverse-transcribed using a mix of random decamers (Ambion) and anchored oligo $(\mathrm{dT})_{20}$ primers (Invitrogen). Real-time PCR was performed using the SyBr Fast kit (Kapa Biosystems) according to the manufacturer's recommendations in a Rotor Gene-6000 instrument (Corbett Research). Expression levels of $t b a-1$ were normalized to the Phe spike RNA and compared with the untreated $t b a-1$ expression level. Fold differences were calculated using the $\Delta \Delta \mathrm{C}_{\mathrm{t}}$ method (Pfaffl, 2001).

\section{Western blot analysis}

Fifty to 100 worms were lysed directly in Laemmli buffer and subjected to SDS-PAGE, transferred onto PVDF membranes, blocked with 5\% nonfat dry milk in Tris-buffered saline containing $0.05 \%$ Tween 20 , and incubated with primary antibodies as indicated. Antibodies used were rabbit CREB Ser133 (Cell Signaling Technology, 1:1000 dilution), mouse actin (Millipore, 1:2000 dilution), and mouse FLAG (Sigma-Aldrich, 1:1000 dilution). Primary antibodies were detected with appropriate HRPconjugated secondary antibodies (Jackson ImmunoResearch Laboratories, 1:5000 dilution).

\section{Tissue-specific mRNA isolation}

Neuronal mRNA was isolated as described previously (Von Stetina et al., 2007) with some modifications. After the different treatments, polyAbinding protein (PAB-1::FLAG)-expressing worms were collected and washed, and RNA-protein complexes were stabilized by incubating worms in $5 \mathrm{ml}$ of $0.5 \%$ formaldehyde in CTX buffer ( $25 \mathrm{~mm}$ K-phosphate, $\mathrm{pH} 6.0$, $1 \mathrm{mM} \mathrm{CaCl}_{2}$, and $1 \mathrm{~mm} \mathrm{MgSO}_{4}$ ) for $1 \mathrm{~h}$ at $4^{\circ} \mathrm{C}$, washed once with CTX, washed twice with $700 \mu$ l of homogenization buffer [HB; $300 \mathrm{~mm} \mathrm{NaCl}$, 50 mм HEPES buffer, pH 7.6, 10 mм $\mathrm{MgCl}_{2}, 1$ mм EGTA, 30 mм EDTA, $0.2 \mathrm{mg} / \mathrm{ml}$ heparin, $10 \%$ glycerol, $1 \mathrm{~mm}$ dithiothreitol, $8 \mathrm{~mm}$ vanadyl 
ribonucleoside complex, $50 \mathrm{U} / \mathrm{ml}$ Rnasin ribonuclease inhibitor (Promega), and EDTA-free protease inhibitor mixture (Roche)], and shockfrozen in liquid nitrogen. The homogenization was done in a TissueLyzer MM 301 Ball Mill Homogenizer (Retsch) three times for $30 \mathrm{~s}$ at $30 \mathrm{~Hz}$. The homogenate was cleaned with centrifugation, and immunoprecipitation was done with $30 \mu \mathrm{l}$ of anti-FLAG M2 agarose bead slurry (SigmaAldrich) equilibrated in HB. Precipitated mRNA-protein complexes were eluted in elution buffer (50 mM Tris- $\mathrm{HCl}, \mathrm{pH}$ 8.0, $10 \mathrm{~mm}$ EDTA, $1.3 \%$ SDS, and $160 \mathrm{U} / \mathrm{ml}$ Rnasin ribonuclease inhibitor), and RNA was isolated using the Direct-zol RNA MiniPrep kit (Zymo Research Cooperation) according to the manufacturer's protocol. The RNA was eluted and concentrated using the RNA Clean \& Concentrator-5 kit (Zymo Research Cooperation) without DNase treatment according to the manufacturer's recommendations.

RNA amplification for the microarrays

Seventy-five nanograms of target RNA were processed using the GeneChip 3' IVT Express kit (Affymetrix) according to the manufacturer's recommendations. Briefly, the RNA was reverse transcribed using oligo(dT) primer. After second-strand cDNA synthesis, the biotin-labeled RNA was purified using magnetic beads; fragmented and hybridized on a GeneChip C. elegans Genome Array (Affymetrix); stained and washed with the FS450_000 fluidics protocol on the GeneChipFluidics Station 450, using the GeneChip Hybridization, Wash, and Stain kit (Affymetrix); and scanned using the GeneChipScanner (Affymetrix). The replicates were processed in three independent batches.

\section{Microarray data analysis}

Preprocessing. Expression data were processed using the affy R package (Gautier et al., 2004). Expression intensities were normalized using the RMA algorithm. Above background expression filtering was applied using the Mas5Calls algorithm: probe sets called as absent (cutoff, $p \geq 0.05$ ) in all arrays of an experiment were excluded, yielding a total of 15,671 and 14,979 probe sets for the temporal profiling study and second experiment, respectively.

Temporal profiling. To characterize temporal profiles after conditioning, probe sets were selected based on differential expression (DE) analysis between naive state and conditioning time points. DE analysis was performed using linear models, as implemented in the limma $\mathrm{R}$ package (Ritchie et al., 2015), considering batch (3 levels) and condition (11 levels corresponding to the naive, starved, adapted, and eight time points after conditioning). From these models, contrasts were first built to compare each conditioning time point with the naive state; $p$ values were corrected for multiple testing (global correction for all probe sets and eight contrasts) using Benjamini and Hochberg false discovery rate (FDR). A probe set was considered as differentially expressed after aversive conditioning if at least one significant contrast was detected (FDR $<0.05$ ). To further limit the number of probe sets entering clustering analysis, a fold change (FC) criteria $\geq|1.8|$ was applied that corresponded to the median $|\mathrm{FC}|$ observed for all significant contrasts. To circumvent overly conservative multiple correction for the control conditions, change after starvation or adaptation (SA) response compared with the naive state was based on FC criteria solely $(\geq|1.8|)$; thus a total of 736 probe sets were identified as responding after SA. Among those, 639 probe sets simultaneously showed differential expression after conditioning and were referred to as SA set.

Before clustering analysis, expression intensities were adjusted for batch effects using R package PamR (Hastie et al., 2014). Hierarchical clustering (average linkage with Pearson's correlation distance) was applied to per-probe mean intensities centered across the 11 states $(\mathrm{N}, \mathrm{S}, \mathrm{A}$, 0 h, 0.5 h, 1 h, 2 h, 4 h, 8 h, 16 h, 24 h).

Cluster assignment was obtained using the dynamic tree cut algorithm implemented in the dynamicTreeCut R package (Langfelder et al., 2008), with a minimum cluster size of 200 .

Second differential expression analysis. Statistical analysis was based on limma linear models including batch (three levels) and condition (seven levels: naive, starved $4 \mathrm{~h}$, starved $8 \mathrm{~h}$, adapted $4 \mathrm{~h}$, adapted $8 \mathrm{~h}$, conditioned $4 \mathrm{~h}$, conditioned $8 \mathrm{~h}$ ). To increase statistical power, differential expression contrasts were built considering combined time points within each state $(4 h+8 h \div 2)$. Thus, the following three contrasts were evaluated: naive versus conditioning $(4 h+8 h)$, starvation $(4 h+8 h)$ versus conditioning $(4 h+8 h)$, and adaptation $(4 h+8 h)$ versus conditioning $(4 \mathrm{~h}+8 \mathrm{~h})$. Association $p$ values were adjusted for multiple testing using the Benjamini and Hochberg FDR method (global correction for all probe sets and all contrasts).

Enrichment analyses. Affymetrix probe set gene annotation was performed using WormBase (www.wormbase.org) database WS245 (affy oligo mapping information). A total of 12,999 mapped genes were thus used as background in all enrichment analyses.

Gene ontology (GO) enrichment analysis was performed using the DAVID functional annotation on-line tool (https://david.ncifcrf.gov/; Huang da et al., 2009). Positive olfactory gene lists corresponding to CREBdependent upregulated, CREB-dependent downregulated, CREB-independent upregulated, and CREB-independent downregulated categories were obtained from published materials in the study by Lakhina et al. (2015; genes identified by SAM analysis reported in Tables S4 and S5 from original report) and annotated using WormBase database WS245. Genes overlapping with our background genes list $(n=12,999)$ were considered for analysis. Given an observed overlap between the previously defined gene categories, we further restricted the analysis to genes exclusively found within one single category, yielding the following nonoverlapping gene lists: CREB-dependent upregulated ( $n=538)$, CREB-dependent downregulated $(n=86)$, CREB-independent upregulated $(n=1195)$, and CREB-independent downregulated $(n=851)$. Enrichment analyses for positive olfactory assay gene lists were performed using Fisher's exact test.

\section{Results \\ Temporal transcriptomic profiling after aversive conditioning}

Previously, we demonstrated that both transcription and translation are required to maintain LTAM after aversive olfactory conditioning (Vukojevic et al., 2012). To gain insight on the gene regulation process during LTAM, we first performed a time assay to define the time points of gene activation related to aversive olfactory LTAM. Therefore, we performed actinomycin D pulse treatment of DA-conditioned worms at different times after training and tested the LTAM of these animals $24 \mathrm{~h}$ after conditioning (Fig. 1a). To define the duration of the pulse treatment, we analyzed the relative amount of tubulin mRNA using realtime PCR. We found that a 5 min actinomycin D treatment inhibited transcription for $1 \mathrm{~h}$ starting $20 \mathrm{~min}$ after treatment (Fig. $1 b)$. Inhibition of transcription using short actinomycin D treatment immediately after conditioning very efficiently blocked LTAM, whereas application of actinomycin D between 45 min and $2 \mathrm{~h}$ after conditioning was not effective. We observed a second peak of inhibition $3 \mathrm{~h}$ after conditioning that corresponds to a wave of gene activation between 3 and $4 \mathrm{~h}$ after conditioning. Thus, our results show that aversive associative LTAM induces a biphasic gene activation with a peak corresponding to immediate genes and a second peak $4 \mathrm{~h}$ after conditioning.

To identify neuronal genes regulated by aversive LTAM in a temporal manner, we used the previously established and characterized pan-neuronal expressed PAB-1::FLAG system (Von Stetina et al., 2007). First, we tested the enrichment of neuronal genes using PAB-1::FLAG-RNA complex purification and tested enrichment of specific genes over total RNA. We tested the levels of known neuronal, or as a control, muscle expressed gene with qPCR in the purified PAB-1-bound RNA pool from pan-neuronal expressed PAB-1::FLAG (Von Stetina et al., 2007) or as a control from myo-3-driven PAB-1::FLAG (Roy et al., 2002). We found that PAB-1::FLAG precipitation enriched the tested RNAs according to their expected tissue specificity (Fig. 1c).

Thus for the microarray study, we collected neuronal enriched mRNA pools using the pan-neuronal promoter-driven FLAG:: 
a

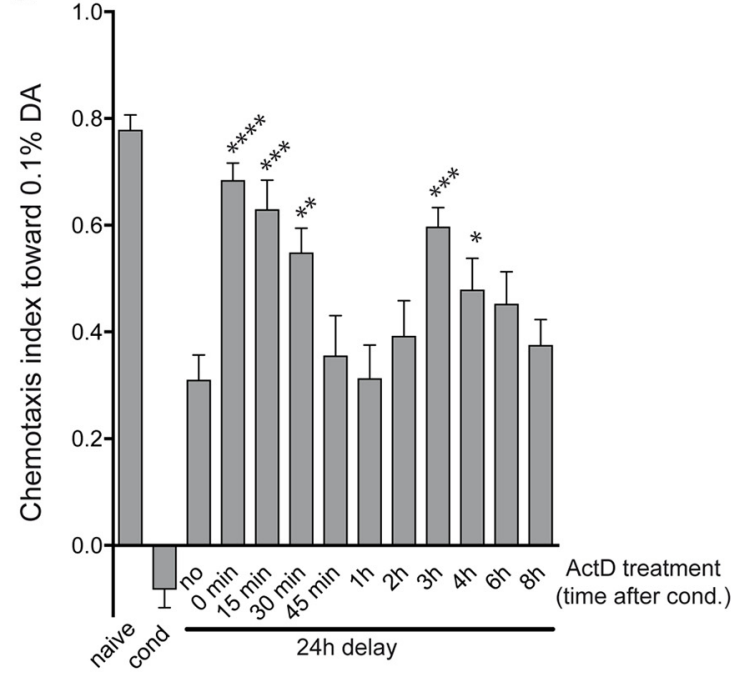

C b

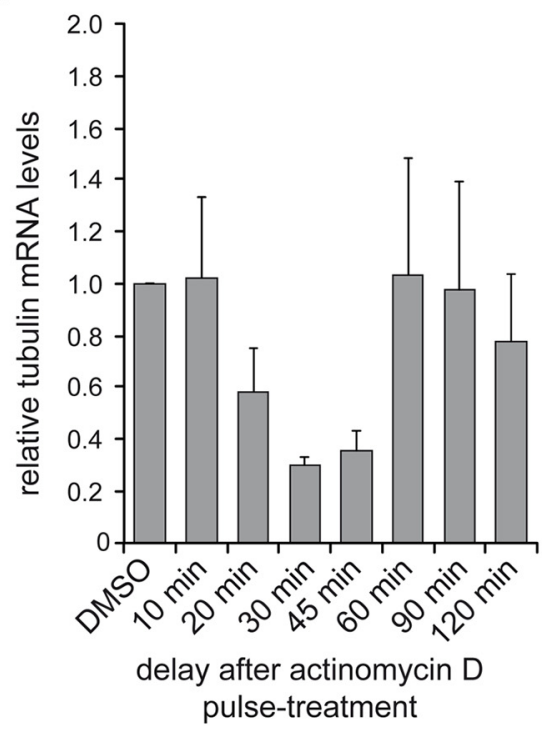

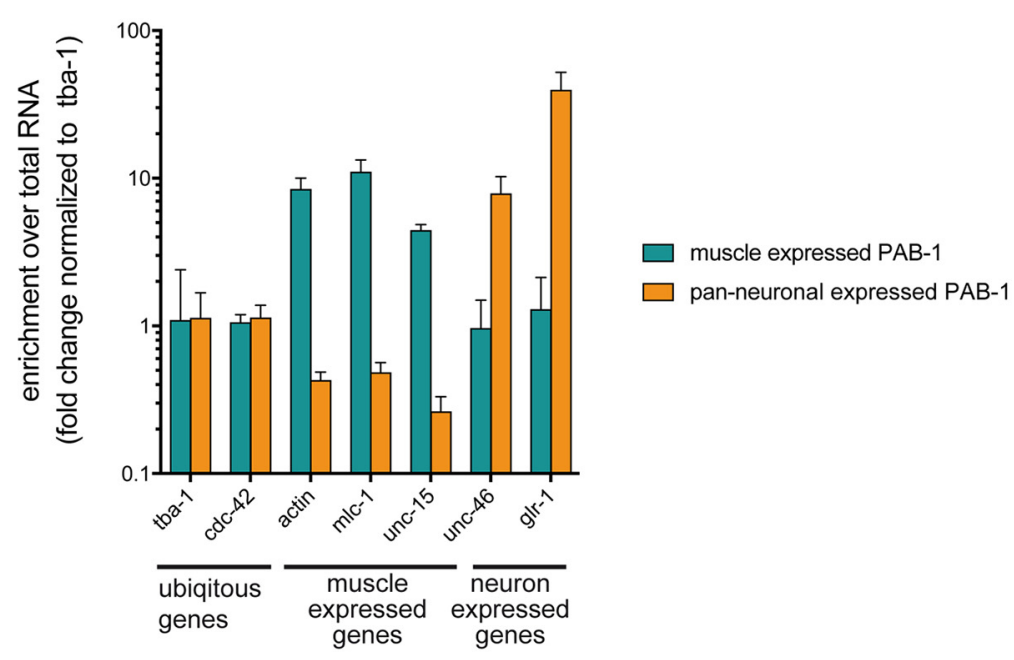

Figure 1. Pulse treatment of actinomycin D shows a biphasic effect on LTAM. $\boldsymbol{a}$, Young adult worms were treated for 5 min with DMSO or actinomycin D in DMSO at different time points after conditioning, and LTAM was tested $24 \mathrm{~h}$ after conditioning. The chemotaxis index for each treatment was calculated as described previously (Bargmann et al., 1993). The graph represents mean and SE. $n=6-16$. Statistical significance was assessed with one-way ANOVA and Dunnett's post hoc test. Treatment conditions were compared with the untreated worms' $24 \mathrm{~h}$ memory. ${ }^{* * * *} p<$ $0.0001 ;{ }^{* *} p<0.001 ;{ }^{* *} p<0.01 ;{ }^{*} p<0.05 . \boldsymbol{b}$, Effect of the actinomycin D on RNA transcription was tested by measuring the RNA levels of tba- 1 transcripts at different time points after 5 min pulse treatment with actinomycin D. tba- 7 mRNA levels were normalized against bacterial spike RNA added to the isolated total RNA before reverse transcription reaction. The graph represents mean and SE of three independent experiments with three technical replicates each. $\boldsymbol{c}$, Enrichment of ubiquitous, neuronal, or muscle-specific genes after PAB-1-mRNA complex purification using tissue-specific expression of PAB-1. The specific gene abundance was normalized to tba- 1 mRNA levels, and enrichment was expressed as fold change compared with the levels in total RNA. The graph represents mean and SE of $4-10$ replicates.

PAB-1 protein (Von Stetina et al., 2007) at different time points after aversive conditioning (Fig. 2a). In addition, we assessed gene expression in untrained worms or animals exposed to starvation or to DA alone. Gene expression profiling for each treatment was performed in three independent biological replicates. First, we analyzed the global gene expression intensity profile using principal component analysis that showed a clear separation between the naive and control states and the different conditioning time points displayed in a regular and chronologically consistent pattern (Fig. $2 b$ ). Using the microarray results, we also tested the global enrichment of neuronal genes in the PAB-1:: FLAG immunoprecipitations by comparing the detected genes with the recent results of Kaletsky et al. (2016) reporting a set of $\sim 11,000$ genes expressed in C. elegans adult neurons. Using this gene expression annotation, we observed a significant increase in the proportion of neuronal expressed genes among genes ex- pressed above background intensity in our microarray study $(72.8 \%)$ compared with the entire set of genes mapped by the array (59\%; proportion test, $p<2.2 \mathrm{e}^{-16}$ ). Although we could show neuronal gene enrichment using pan-neuronal expressed PAB-1 over the total RNA pool, neither the qPCR experiments nor the global enrichment analysis fully rule out alterations in the PAB-1 expression across different neuronal cells.

Next, to characterize transcriptomic patterns induced by conditioning, we first assessed differential expression between each conditioning time point and naive state (see Materials and Methods). Using limma-moderated $t$ tests, we selected a total of 3563 transcripts exhibiting at least one time point with significant contrast (FDR, <0.05; fold change, $\geq|1.8|$; Fig. $2 c$ ).

Among the 3563 identified transcripts, 639 probe sets also showed change between naive state and adaptation [DA only, conditioned stimulus (CS)] or starvation [food withdrawal only, 
a
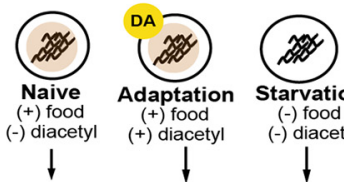

Starvation Conditioning $(-)$ food $(-)$ food (+) diacetyl diacety

$\downarrow$ $\stackrel{012 \quad 4 \quad 8 \quad 16 \quad 24 h}{\longrightarrow}$ Neuronal enriched RNA extraction

C

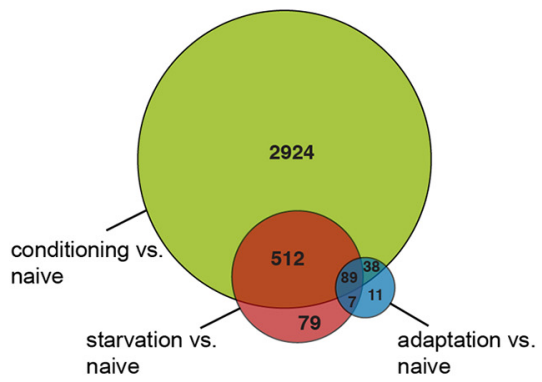

d
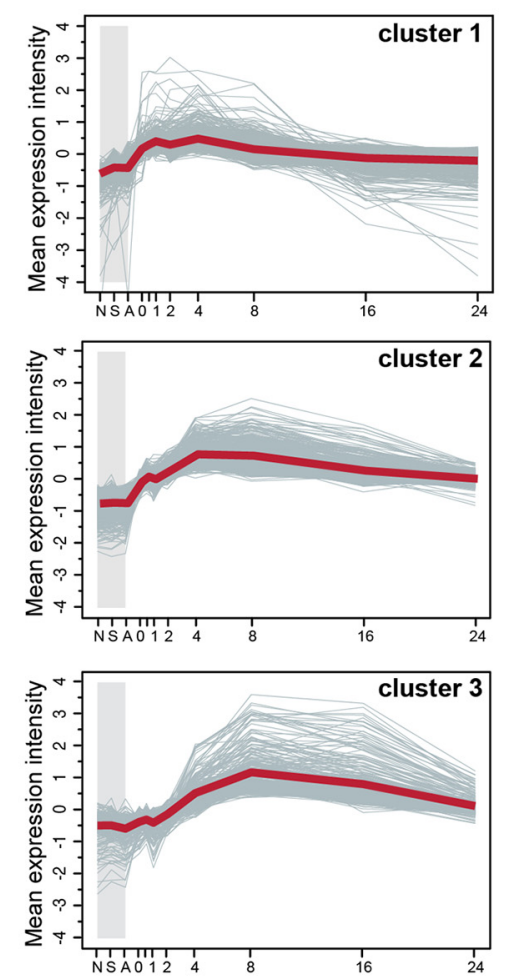

b

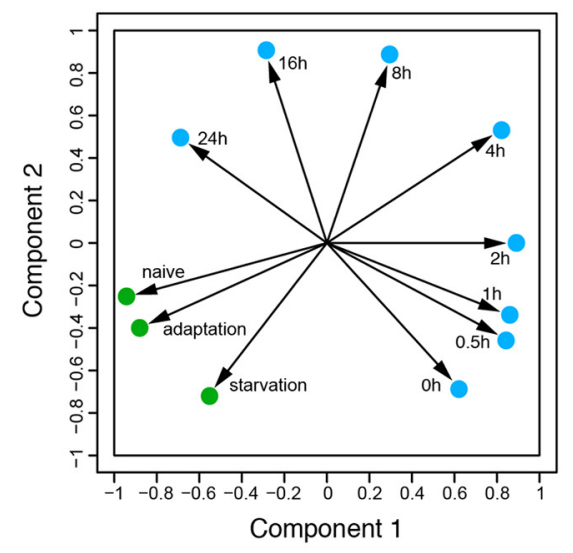

e

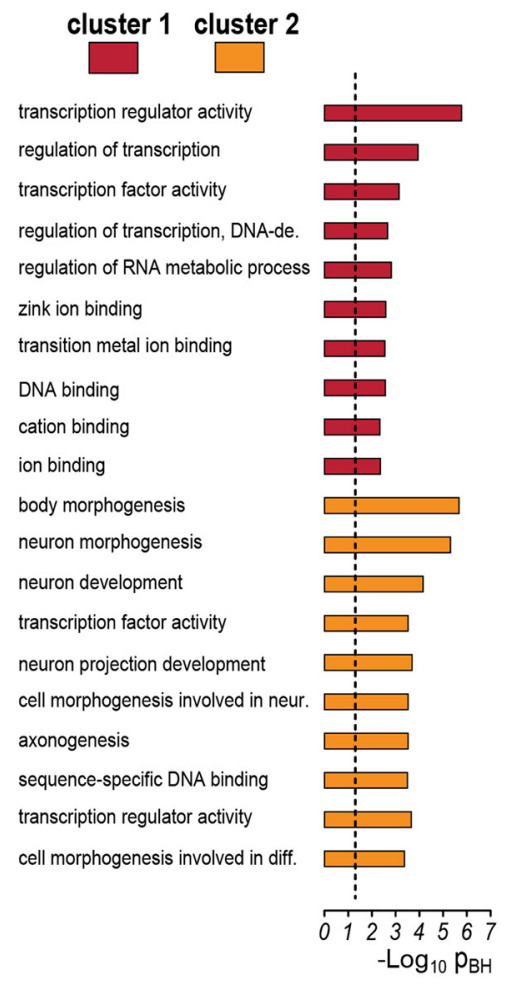

Figure 2. Temporal transcriptomic profiling after aversive conditioning. $\boldsymbol{a}$, Neuronal enriched RNA was extracted from naive worms, DA-adapted worms, and starved worms directly after $1 \mathrm{~h}$ treatment or at eight time points after three rounds of $1 \mathrm{~h}$ aversive conditioning training sessions each. $\boldsymbol{b}$, Principal component analysis (PCA) result of transcriptomic profiles. PCA was performed on genome-wide, probe-centered average intensities per state. The first two components obtained are represented. $c$, Differential expression was assessed between each conditioning time point and naive state ( $F D R,<0.05 ;|F C| \geq 1.8$ ), yielding a total of 3563 probe sets differentially expressed between conditioning and naive state (green circle), among which 639 probe sets also exhibited change between naive and adaptation or starvation states ( $|\mathrm{FC}| \geq 1.8)$. d, Upregulation clusters identified by hierarchical clustering analysis of differentially expressed probe sets (clusters $1-3$ ); naive $(\mathrm{N})$, starvation $(S)$, adaptation $(A)$, and conditioning time points $(0,0.5,1,2,4,8,16$, and $24 \mathrm{~h})$ are represented along the horizontal axis; the vertical axis indicates probe-centered average

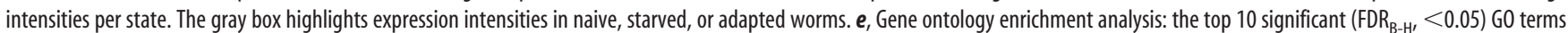
(molecular function, biological process) are displayed for each upregulation cluster (no significant $\mathrm{G} 0$ term was identified for cluster 3 ).

unconditioned stimulus (US); fold change, $\geq|1.8|$ ] (Fig. 2c). These probe sets were referred to as SA-regulated probe sets.

To gain insight on the temporal patterns of expression after conditioning, we performed hierarchical clustering on expression intensities of the 3563 identified transcripts across the 11 states (see Materials and Methods) and thus identified six distinct patterns of regulation.

Three of the identified clusters (clusters 1-3) corresponded to transcripts upregulated after conditioning (Fig. $2 d$ ). These clus- ters encompassed only a minor fraction of SA probe sets (Fig. 3a), suggesting that the observed transcriptional patterns may not reflect early adaptation- or starvation-dependent transcriptional responses.

Among those, clusters 1 and 2 showed temporal patterns that closely coincided with kinetics of gene activation during LTAM determined after actinomycin D treatment (Fig. 1). Interestingly, both clusters 1 and 2 were characterized by activation immediately after conditioning (Fig. $2 d$ ), which in the case of cluster 1 

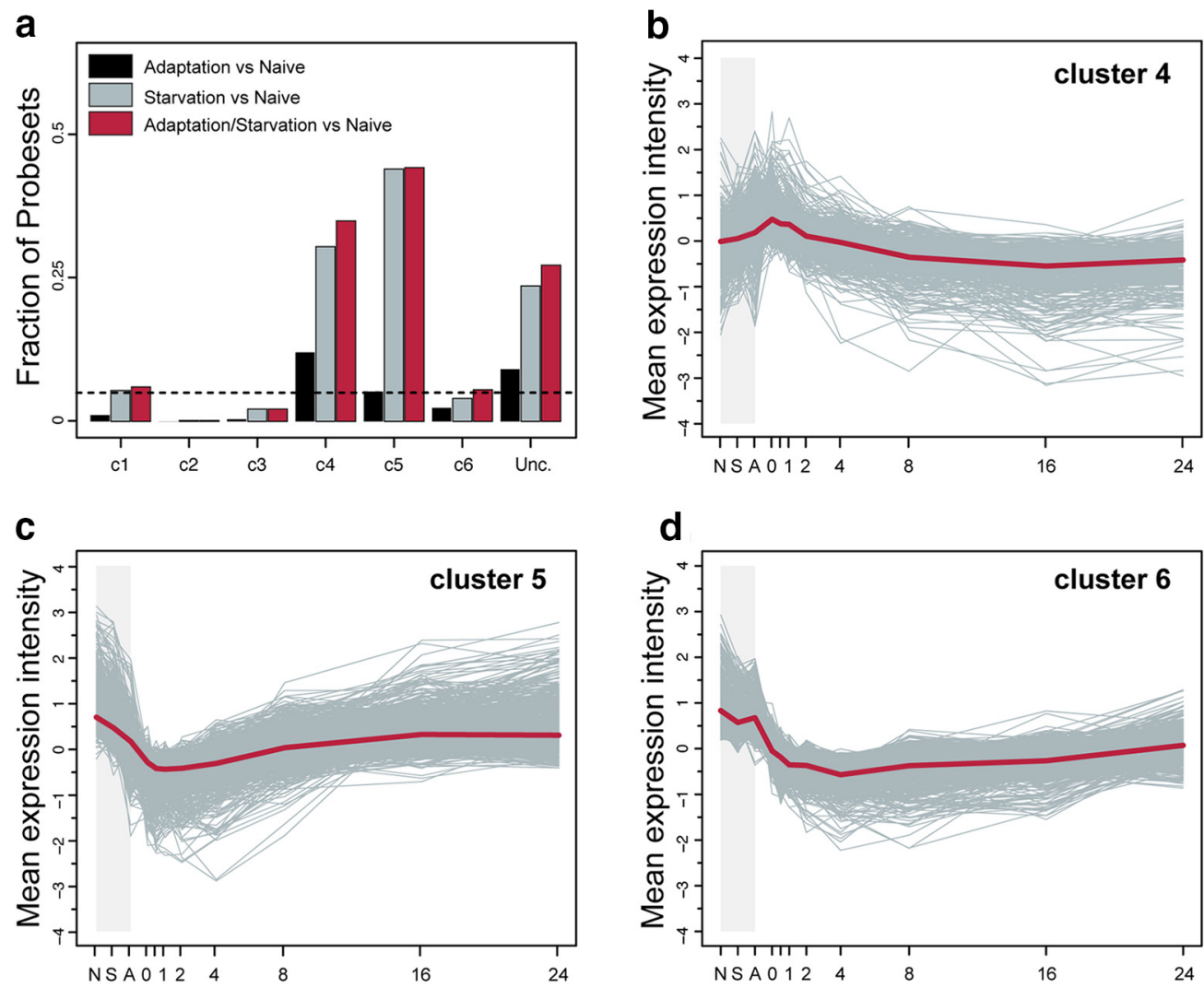

Figure 3. Differential expressions after starvation and adaptation across aversive conditioning clusters and temporal expression profiles for clusters $4-6 . \boldsymbol{a}$, Temporal expression profile clusters are represented along the horizontal axis. Vertical bars indicate the fraction of transcripts exhibiting suggestive differential expression (FC threshold, $\geq|1.8|$ ) between naive and adaptation (black bar), naive and starvation (gray bar), and naive and adaptation or starvation (red bar; $S A$ set). The horizontal dashed line represents the 0.05 fraction. Unc., Unclassified probe sets. $\boldsymbol{b}$ - $\boldsymbol{d}$, Clustering of temporal expression profiles of genes downregulated after aversive conditioning (clusters $4-6$ ). Clusters resulting from hierarchical clustering analysis of expression intensities of the $n=3563$ transcripts differentially expressed after aversive conditioning (cluster $4, n=423$; cluster $5, n=883$; cluster $6, n=723 ; 55$ unclassified transcripts not shown). Naive (N), starvation (S), adaptation (A), and conditioning time points $(0,0.5,1,2,4,8,16$, and $24 \mathrm{~h})$ are represented along the horizontal axis; the vertical axis indicates probe-centered average intensities per state.

remained elevated for at least $4 \mathrm{~h}$ after conditioning. On the other hand, genes in cluster 2 showed a biphasic activation pattern with an immediate minor activation peak followed by a dominant upregulation peak culminating $\sim 4 \mathrm{~h}$ after conditioning (Fig. $2 d$ ), which coincide with the second wave of transcription required for LTAM formation (Fig. 1). GO analysis (see Materials and Methods) revealed enrichment for transcriptional activity-related terms $\left(\mathrm{FDR}_{\mathrm{B}-\mathrm{H}},<0.05\right)$ in clusters 1 and 2 (Fig. 2e). Cluster 2 also showed over-representation of genes linked to neuronal development and neuronal function (Fig. 2e). Finally, the last upregulation cluster 3 contained genes reaching the maximum activation $\sim 8 \mathrm{~h}$ after conditioning. However, no specific GO term enrichment was observed in this cluster. The three remaining clusters (clusters 4-6) contain a substantial fraction of genes that are characterized by an early or a delayed downregulation pattern after conditioning (Fig. $3 b-d$ ). These gene clusters showed significant enrichment $\left(\mathrm{FDR}_{\mathrm{B}-\mathrm{H}},<0.05\right)$ for $\mathrm{GO}$ categories broadly related to metabolic activity (Table 1). In addition, two of these clusters (clusters 4 and 5) encompassed a majority of the SA probe sets (Fig. $3 a$ ), indicating that the observed transcriptional patterns may primarily coincide with adaptation- or starvationrelated transcriptomic response. Interestingly, among the clusters containing downregulation genes, cluster 5 showed a significant over-representation of genes related to neuropeptide signaling pathways $\left(p=5.6 \mathrm{e}^{-15}\right)$ and notably contained 17 FMRF amide (Phe-Met-Arg-Phe- $\mathrm{NH}_{2}$ )-like peptides, 13 nlp peptides, and 10 insulin-like peptides (Table 2). Finally, in the case of cluster $c 6$, we observed only a minor fraction of the early SA probe sets (Fig. 3a), suggesting that starvation and adaptation response may also induce delayed transcriptional downregulation change. Altogether, temporal kinetic analysis of LTAM-regulated genes identified three waves of upregulation events, with peaks occurring at 1,4 , and $8 \mathrm{~h}$ after conditioning. Similarly, nearly half of the identified genes were found to be downregulated with kinetic patterns similar to the upregulated ones. However, these genes are more likely linked to metabolic processes, suggesting that they may reflect gene expression changes attributable to general homeostatic changes.

In a second independent experiment, we included additional conditional filters to differentiate between memory-regulated and starvation-or adaptation-related genes. We focused on genes differentially expressed at 4 and $8 \mathrm{~h}$ after conditioning as they represent ideal time points to cover most LTAM genes (Fig. 2). We assessed specificity of aversive LTAM-regulated transcriptional change ( 4 and $8 \mathrm{~h}$ ) compared with adaptation- and starvation-regulated delayed responses (Fig. 4a). Transcriptomic profiles were obtained from neuronal enriched RNA extracted from naive worms, 4 and $8 \mathrm{~h}$ after conditioning, starvation (US only), and adaptation (CS only). Differential expression was subsequently evaluated between naive and conditioning states, starvation and conditioning states, and adaptation and conditioning states using limma-moderated $t$ tests. To increase statistical power in differential expression analysis, contrasts were built considering the combined time points at 4 and $8 \mathrm{~h}$ within each state (see Materials and Methods). 
Table 1. Gene ontology enrichment analysis of aversive conditioning temporal profiling clusters 4-6

\begin{tabular}{|c|c|c|c|c|c|}
\hline$\overline{\text { Cluster }}$ & Category & Term & Count & Fold enrichment & $p$ \\
\hline \multirow[t]{10}{*}{$c 4$} & $C \mathrm{C}$ & G0:0005576 extracellular region & 21 & 3.56 & $1.01 e^{-6}$ \\
\hline & MF & G0:0003796 lysozyme activity & 5 & 21.86 & $3.99 \mathrm{e}^{-5}$ \\
\hline & $\mathrm{BP}$ & G0:0055114 oxidation reduction & 21 & 2.73 & $6.28 e^{-5}$ \\
\hline & BP & G0:0006631 fatty acid metabolic process & 8 & 7.15 & 0.000101223 \\
\hline & $\mathrm{BP}$ & G0:0006026 aminoglycan catabolic process & 5 & 17.12 & 0.000139303 \\
\hline & BP & G0:0000272 polysaccharide catabolic process & 5 & 17.12 & 0.000139303 \\
\hline & BP & G0:0008340 determination of adult life span & 16 & 2.95 & 0.000288077 \\
\hline & $\mathrm{BP}$ & G0:0010259 multicellular organismal aging & 16 & 2.95 & 0.000288077 \\
\hline & BP & G0:0007568 aging & 16 & 2.95 & 0.000288077 \\
\hline & MF & G0:0004175 endopeptidase activity & 15 & 3.07 & 0.00030769 \\
\hline \multirow[t]{10}{*}{$c 5$} & $\mathrm{BP}$ & G0:0007218 neuropeptide signaling pathway & 21 & 8.92 & $5.60 e^{-15}$ \\
\hline & $\mathrm{BP}$ & G0:0007186 G-protein-coupled receptor protein signaling pathway & 34 & 3.03 & $1.18 \mathrm{e}^{-8}$ \\
\hline & BP & G0:0030258 lipid modification & 17 & 5.32 & $4.05 \mathrm{e}^{-8}$ \\
\hline & BP & G0:0016054 organic acid catabolic process & 13 & 6.34 & $3.08 \mathrm{e}^{-7}$ \\
\hline & $\mathrm{BP}$ & G0:0046395 carboxylic acid catabolic process & 13 & 6.34 & $3.08 \mathrm{e}^{-7}$ \\
\hline & MF & G0:0005179 hormone activity & 12 & 6.84 & $3.63 \mathrm{e}^{-7}$ \\
\hline & $\mathrm{CC}$ & G0:0005576 extracellular region & 40 & 2.32 & $6.02 \mathrm{e}^{-7}$ \\
\hline & $\mathrm{BP}$ & G0:0030259 lipid glycosylation & 13 & 5.82 & $8.82 \mathrm{e}^{-7}$ \\
\hline & $\mathrm{BP}$ & G0:0007166 cell-surface receptor-linked signal transduction & 35 & 2.27 & $8.29 \mathrm{e}^{-6}$ \\
\hline & $\mathrm{BP}$ & G0:0055114 oxidation reduction & 40 & 2.10 & $1.05 \mathrm{e}^{-5}$ \\
\hline \multirow[t]{10}{*}{ c6 } & MF & G0:0042302 structural constituent of cuticle & 42 & 7.13 & $9.51 e^{-26}$ \\
\hline & $\mathrm{BP}$ & G0:0006091 generation of precursor metabolites and energy & 35 & 5.51 & $7.26 e^{-17}$ \\
\hline & $\mathrm{BP}$ & G0:0006006 glucose metabolic process & 21 & 8.70 & $1.57 e^{-14}$ \\
\hline & MF & G0:0005198 structural molecule activity & 59 & 2.79 & $2.25 \mathrm{e}^{-13}$ \\
\hline & $\mathrm{BP}$ & G0:0019320 hexose catabolic process & 17 & 10.09 & $4.69 e^{-13}$ \\
\hline & BP & G0:0006007 glucose catabolic process & 17 & 10.09 & $4.69 e^{-13}$ \\
\hline & $\mathrm{BP}$ & G0:0046365 monosaccharide catabolic process & 17 & 10.09 & $4.69 e^{-13}$ \\
\hline & BP & G0:0006096 glycolysis & 15 & 12.14 & $4.87 e^{-13}$ \\
\hline & $\mathrm{BP}$ & G0:0019318 hexose metabolic process & 21 & 6.80 & $4.42 e^{-12}$ \\
\hline & $\mathrm{BP}$ & G0:0046164 alcohol catabolic process & 17 & 8.90 & $5.78 e^{-12}$ \\
\hline
\end{tabular}

Only the top 10 enriched terms per cluster are listed. CC, Cellular component; MF, molecular function; BP, biological process.

Table 2. Neuropeptide genes identified in starvation-related cluster 5

\begin{tabular}{|c|c|}
\hline Category & Genes \\
\hline $\begin{array}{l}\text { FMRF amide-like peptides } \\
\text { (Phe-Met-Arg-Phe--NH }{ }_{2} \text { ) }\end{array}$ & $\begin{array}{l}\text { flp-1, flp-11, flp-13, flp-16, flp-17, flp-18, flp-19, flp-21, flp-22, } \\
\quad \text { flp-24, flp-3, flp-4, flp-5, flp-6, flp-7, flp-8, flp-9 }\end{array}$ \\
\hline Neuropeptides & $\begin{array}{l}n l p-10, n / p-11, n / p-15, \text { nlp-18, nlp-21, nlp-3, nlp-35, nlp-40, } \\
\quad \text { nlp-42, nlp-47, nlp-5, nlp-6, nlp-7 }\end{array}$ \\
\hline Insulin-like peptides & $\begin{array}{l}\text { ins- } 1 \text {, ins- } 18 \text {, ins- } 22 \text {, ins- } 24 \text {, ins- } 26 \text {, ins- } 3 \text {, ins- } 32 \text {, ins }-33 \text {, ins- } 4 \text {, } \\
\text { ins- } 6\end{array}$ \\
\hline
\end{tabular}

In this second approach, we identified 1039 probe sets (706 upregulated, 333 downregulated) that simultaneously showed significant expression change between conditioning versus naive state, adaptation, and starvation (FDR, <0.05). Thus, these probe sets may represent specific memory-regulated transcripts. Absolute fold changes observed were higher for naive differential expression (median absolute FC, 1.9 across 1039 probe sets) than for adaptation and starvation (median absolute FC, 1.5 and 1.4, respectively).

We next examined the overlap between these memoryregulated probe sets and the set of 3563 probes identified in the first transcriptomic profiling study. This revealed that $82.4 \%$ of the 706 upregulated memory-regulated probe sets were previously identified as being differentially expressed after conditioning and had a similar direction of regulation, whereas only a minor fraction $(2.8 \%)$ showed immediate change after adaptation or starvation (SA set). In contrast, only $55.6 \%$ of the 333 downregulated memory-regulated probe sets were consistently identified in the temporal profiling experiment without showing simultaneous early change in response to starvation or adaptation, whereas $24.9 \%$ showed early change in response to starvation or adaptation (SA set). Thus, this result further indicates that downregulated genes are likely related to starvation or adaptation response.

Finally, we compiled results from both microarray experiments (Fig. $4 b$ ) to generate a specific core set of probes meeting the following criteria: (1) differential expression after conditioning in the temporal profiling study; (2) not included in early SA set; and (3) identified as a memory-regulated gene in the second experimental setup with a direction of regulation consistent with the one observed in the temporal profiling study. Applying these filters, we defined a core set of aversive olfactory LTAM-regulated transcripts, which included a total of 582 upregulated and 185 downregulated probe sets, corresponding to 538 and 174 genes, respectively (Fig. 4c). As expected from our previous results, a majority of this core set was encompassed in cluster 2 (48\%), which also showed the highest specificity toward adaptation and starvation (Fig. $4 d$ ). In summary, we defined a core long-term memory gene set that is mostly upregulated during aversive LTAM.

\section{Different long-term memory paradigms affect transcriptional regulation of mostly identical genes}

By combining positive conditioning assay with genome-wide transcriptional analysis of CREB mutants, Lakhina et al. (2015) recently reported sets of CREB-dependent and -independent LTAM-regulated genes in C. elegans. In this positive olfactory assay, the odorant 2-butanone is repeatedly presented in association with food, which results in formation of an LTAM toward 2-butanone (Kauffman et al., 2011). Previously, positive olfactory associative LTAM was shown to be dependent on CREB activity (Kauffman et al., 2011). To further support that the different LTAMs in C. elegans share common mechanisms, we 

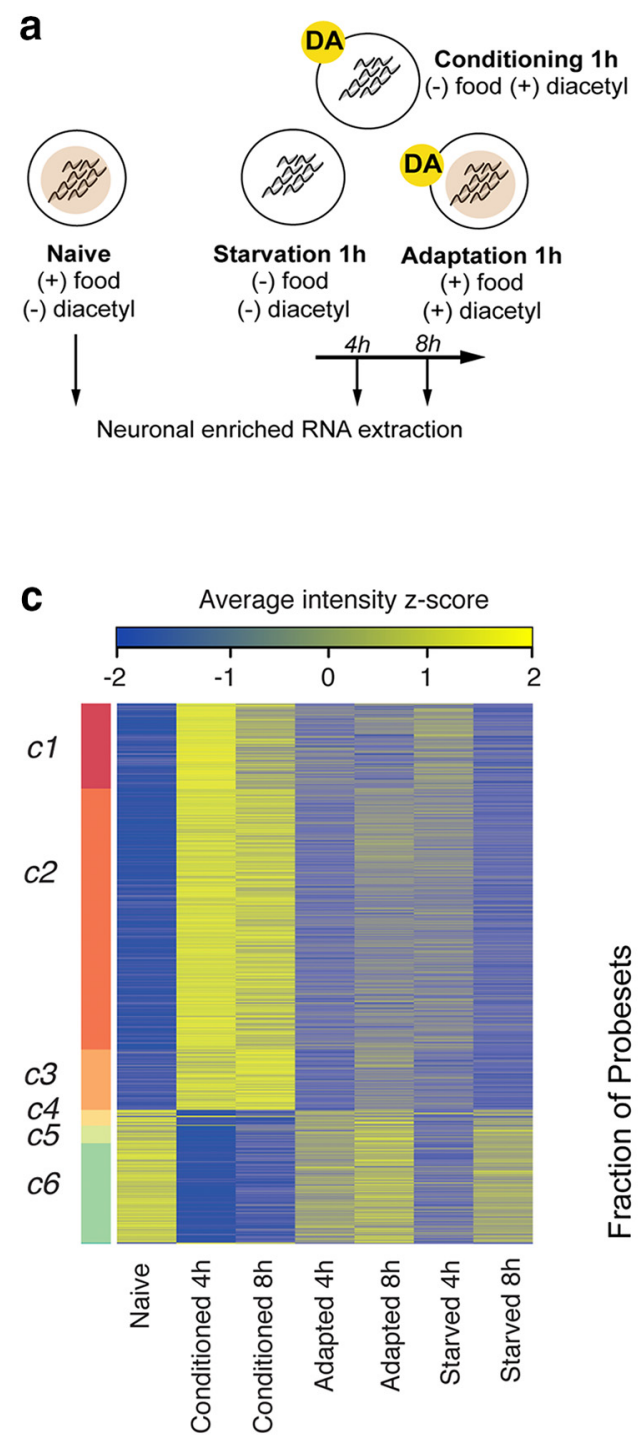

b

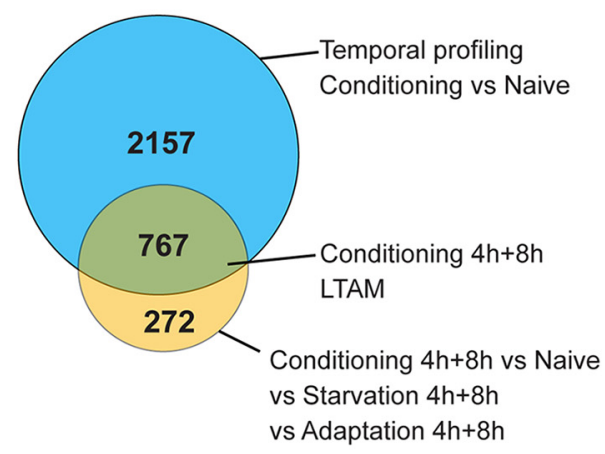

d

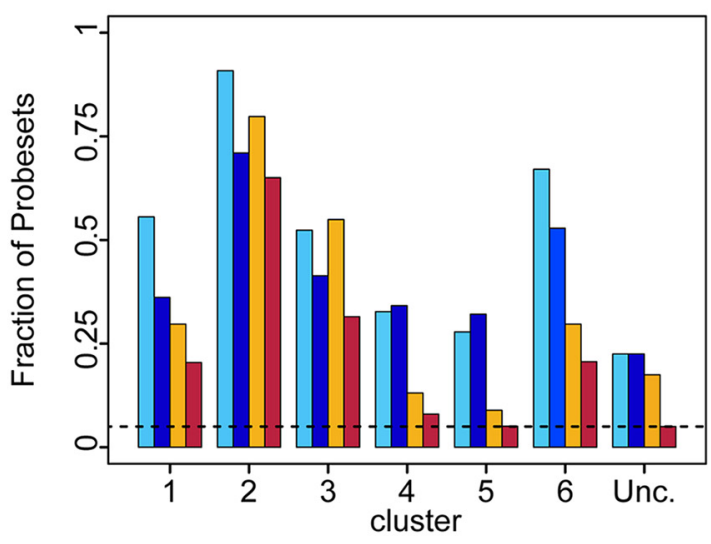

Figure 4. Differential expression between conditioning, starvation, and adaptation delayed ( 4 and $8 \mathrm{~h}$ ) transcriptomic response. $\boldsymbol{a}$, Neuronal enriched RNA was extracted at two time points after aversive conditioning assay ( 4 and $8 \mathrm{~h}$ ) and at two time points after DA adaptation ( 4 and $8 \mathrm{~h}$ ) and starvation ( 4 and $8 \mathrm{~h}$ ). In addition, RNA extraction was performed from naive worms. $\boldsymbol{b}$, Differential expression was assessed between conditioning (combined time points $4 \mathrm{~h}+8 \mathrm{~h}$ ) and naive, starvation (combined time points $4 \mathrm{~h}+8 \mathrm{~h}$ ), and adaptation (combined time points $4 \mathrm{~h}+8 \mathrm{~h}$ ), yielding 1039 probe sets identified. Among those, 767 probe sets overlapped with probe sets identified from the temporal profiling, with a consistent direction of regulation after conditioning across the two experiments. $\boldsymbol{c}$, Heat map of the identified 767 transcripts. The left horizontal colored bar indicates cluster assignment obtained from the temporal profiling experiment. $\boldsymbol{d}$, Fraction of transcripts from the temporal profiling study that were reidentified in the second experiment after the indicated filtering steps. Cluster assignment is represented along the horizontal axis. Unc., Unclassified transcript.

tested the effect of $c r h-1$ deletion on aversive LTAM. We found that deletion of $c r h-1$ impaired aversive LTAM (Fig. $5 a$ ), which is in line with the role of CREB in different types of long-term memory in worms (Kauffman et al., 2011; Timbers and Rankin, 2011; Lakhina et al., 2015). Next, we analyzed the phosphorylation status of $c r h-1$ after aversive LTAM conditioning. Similar to vertebrates, phosphorylation of crh-1 occurs after olfactory LTAM and reflects the activation of the protein (Kauffman et al., 2011). In accordance with our behavioral test result, we could detect an increase in crh-1 phosphorylation during aversive LTAM peaking directly after training. However, an elevated phosphorylation of $c r h-1$ could be detected up to $16 \mathrm{~h}$ after conditioning (Fig. 5b). Finally, we analyzed the temporal requirement for CREB activity. For this, we first generated a crh-1 (lf) mutant transgenic strain carrying the heat-shock promoterdriven wild-type $c r h-1:$ :flag DNA and tested the expression kinetics of CRH-1 after heat shock (Fig. $5 c$ ). Using the FLAG-tag to detect the wild-type CRH- 1 after 30 min of heat shock, we found that the protein is transiently expressed in a narrow time window (Fig. 5c), allowing us to test the temporal requirement for CREB during aversive LTAM. Thus, we performed LTAM tests using our transgenic strain with or without heat shock before, or at different time points after, conditioning. Whereas transient expression of the wild-type $\mathrm{crh}-1$ before conditioning was not able to rescue the crh-1(lf) mutant LTAM defect (data not shown), transient expression of the wild-type protein in $c r h-1(l f)$ worms within the first $2 \mathrm{~h}$ after conditioning was sufficient to partially rescue the $24 \mathrm{~h}$ memory impairment of the mutant worms (Fig. $5 d$ ). On the other hand, later expression of CRH-1 was not sufficient to rescue LTAM (Fig. $5 d$ ). These results suggest that $c r h-1$ activity is necessary within the first $2 \mathrm{~h}$ after conditioning. However, we cannot exclude the possibility that the initial CREBdependent gene activation is a prerequisite for later CREBmediated gene activation processes; thus, the expression of the 
a

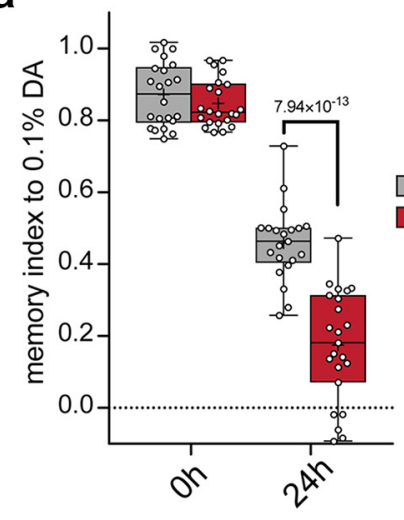

C

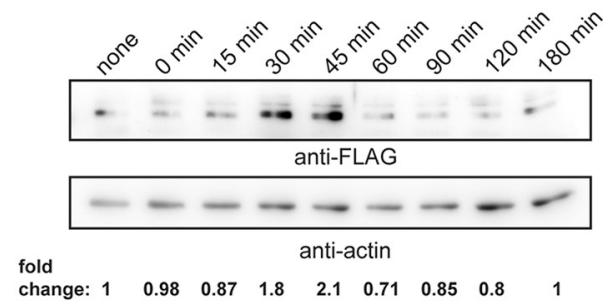

d

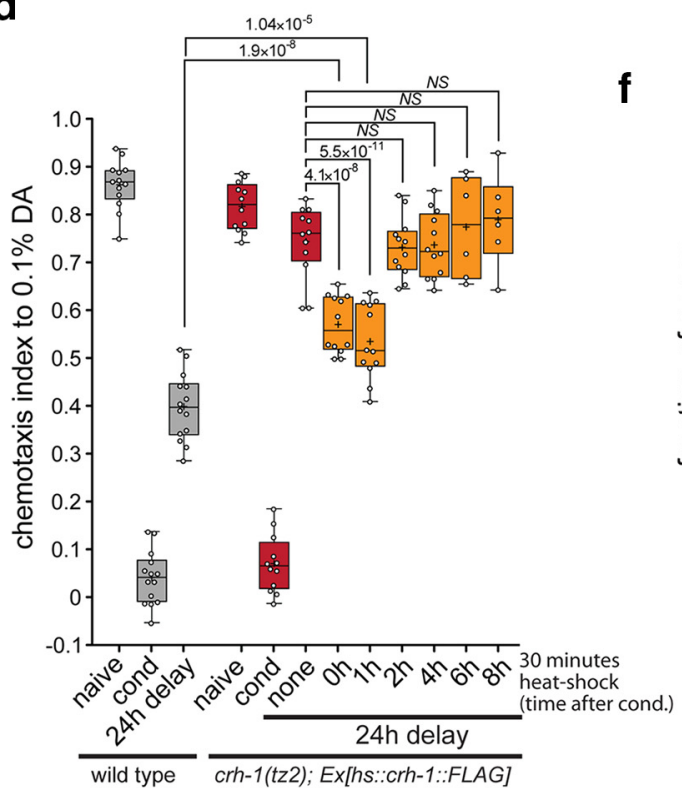

b

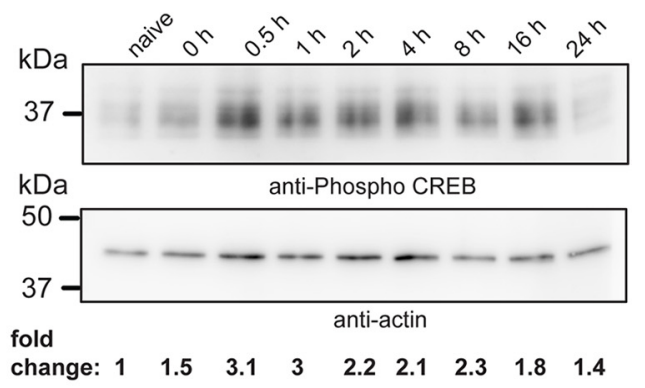

e
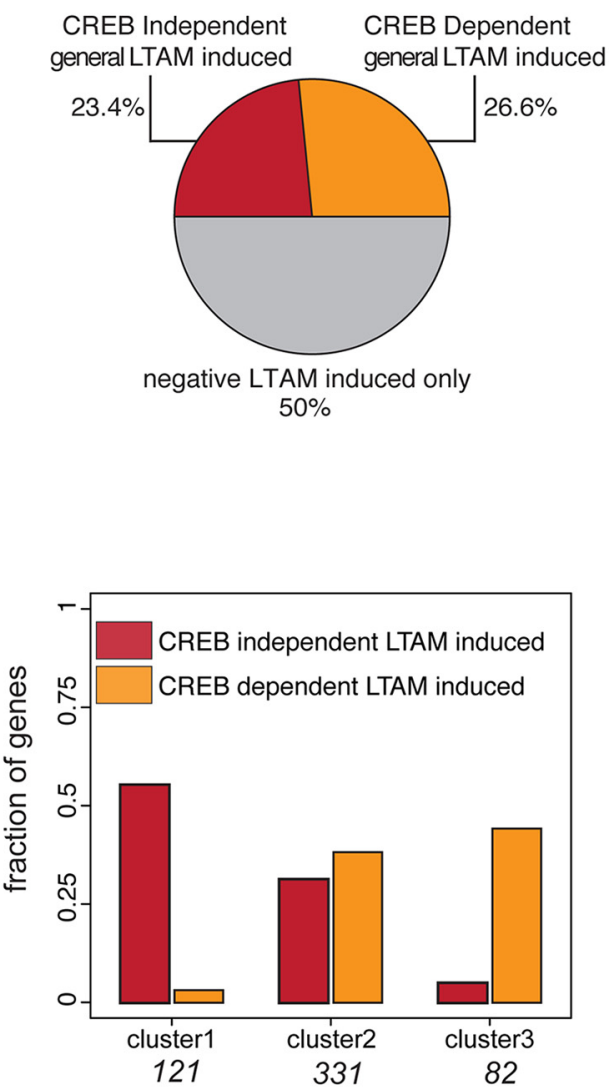

Figure 5. CREB-dependent transcriptional gene activation regulates aversive LTAM. $\boldsymbol{a}$, Role of CREB in aversive LTAM was tested by conditioning wild-type or crh-1 (tz2) mutant worms. Learning was assessed immediately after training $(0 \mathrm{~h}$ ), and LTAM was tested $24 \mathrm{~h}$ after conditioning $(24 \mathrm{~h})$. Box plot and individual data points are presented for each condition and genotype. Statistical significance was assessed with two-way ANOVA (interaction effect: $F_{(1,84)}=30.77, p=3.3 \times 10^{-7}$ ) and post hoc t tests between groups as indicated (Bonferroni's adjusted $p$ values are reported). $\boldsymbol{b}$, Time-dependent CRH-1 phosphorylation after aversive olfactory LTAM was analyzed in total worm lysates with Western blots using Ser133-specific CREB antibody (top). For loading control, membranes were reblotted for actin (bottom). c, Temporal expression of the wild-type CRH-1::FLAG after heat shock induction of the crh-1(tz2), utrEx80[hs:::crh-1::flag, sur-5:::dsRed, myo-3:::rfp] transgenic line. For each condition, lysates from 100 transgenic worms were analyzed using FLAG antibody (top). As the loading control, membranes were reprobed for actin (bottom). This characterized transgenic line was used for the following rescue experiment. $\boldsymbol{d}$, The $c r h-1$ (tz2), utrEx80[hs:::crh-1::flag, sur-5:::dsRed, myo-3::rfp] transgenic animals or wild-type worms were conditioned, and for the temporal rescue, animals were subjected to 30 min heat shock after conditioning as indicated. LTAM was assessed $24 \mathrm{~h}$ after conditioning. Box plot and individual data points are presented for each condition and genotype. Statistical significance was assessed with one-way ANOVA $\left(F_{(11,125)}=214.2, p<10^{-15}\right)$ and post hoc $t$ tests between groups as indicated (Bonferroni's adjusted $p$ values are reported). $\boldsymbol{e}$, Pie chart representing the proportion of LTAM CREB-dependent/independent-induced genes identified after positive conditioning, among the 538 core set of genes upregulated after aversive conditioning. $\boldsymbol{f}$, Distribution of genes classified by Lakhina et al. (2015) as CREB dependent or independent across the aversive olfactory LTAM gene clusters 1-3. The total number of genes in each cluster is indicated along the horizontal axis. 
a

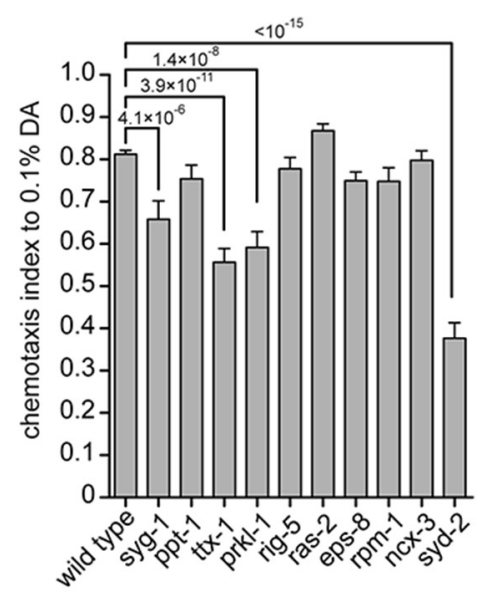

b

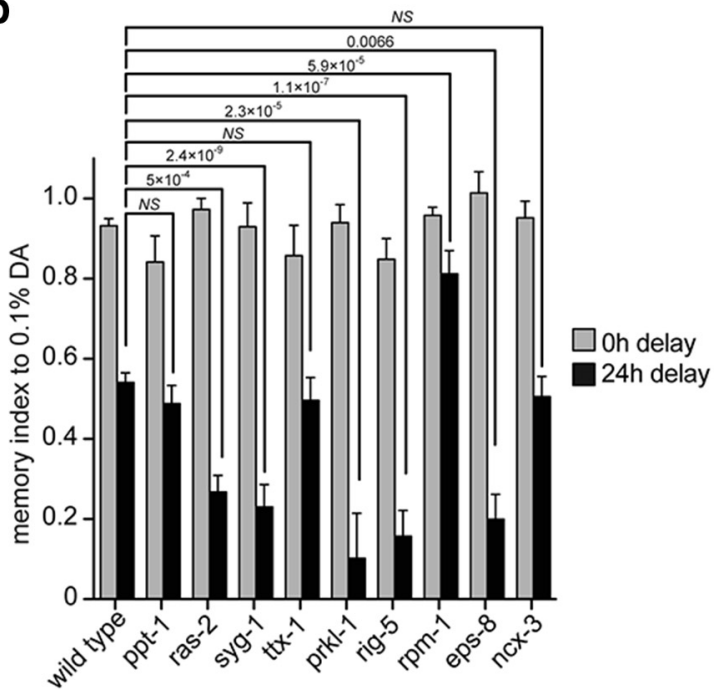

Figure 6. Deletion of the selected LTAM-regulated genes identified by microarray affect aversive LTAM. $\boldsymbol{a}$, Chemotaxis to $0.1 \%$ DA of wild-type or different mutant worms as indicated was tested in synchronized young adult worms. The graph represents mean and SE. $n=9-21$. Statistical significance was assessed with one-way ANOVA $\left(F_{(10,253)}=30.23, p<10^{-15}\right)$ and post hoct tests (Bonferroni's adjusted $p$ values are reported). $\boldsymbol{b}$, To test aversive LTAM, young adult wild-type or different mutant worms were conditioned with DA as described in Materials and Methods. After conditioning, learning was assessed immediately after training ( $0 \mathrm{~h}$, gray bars), and LTAM was tested $24 \mathrm{~h}$ after conditioning $(24 \mathrm{~h}$, black bars). The graph represents mean and SE. $n=7-18$. Statistical significance was assessed with one-way ANOVA (learning: $F_{(9,196)}=1.66, p=0.101$; memory: $F_{(9,192)}=16.58, p<10^{-15}$ ) and post hoc $t$ tests (Bonferroni's adjusted $p$ values are reported).

wild-type protein in $\mathrm{crh}$-1(lf) animals at later time points is not sufficient to rescue LTAM. Altogether, our results show that, like positive olfactory LTAM (Kauffman et al., 2011; Lakhina et al., 2015), aversive olfactory LTAM requires CREB activity and that early CREB activity after conditioning is necessary for aversive LTAM.

To test potential overlap between positive and aversive LTAMinduced genes, we compared our core set of 582 probes that correspond to 538 genes upregulated after aversive conditioning with the set of genes identified as upregulated after positive conditioning, in either a CREB-dependent or an independent manner (Lakhina et al., 2015; see also Materials and Methods). Comparison of the two datasets using different LTAM paradigms demonstrated a significant overlap between the genes identified (Fisher's exact test, $\left.p<1 \mathrm{e}^{-50}\right)$, with $269(50.0 \%$ relative to 538$)$ genes in common (Fig. $5 e$ ). In addition, only six upregulated core memory-regulated genes $(1.1 \%)$ were previously defined as downregulated during positive LTAM, indicating high consistency between the two conditioning paradigms. We also used the CREB-dependency categorization reported by Lakhina et al. (2015) to get an insight on possible common mechanisms, and we observed in our aversive conditioning core set an almost equal representation of genes previously defined as CREB dependent and CREB independent (Lakhina et al., 2015; Fig. 5e). Interestingly, we found differences in the distribution of previously classified CREB-dependent/independent upregulated genes (Lakhina et al., 2015) across the different clusters. Genes classified as CREB independent were enriched in cluster 1 (Fisher's exact test, $p=$ $\left.1.3 \mathrm{e}^{-15}\right)$, cluster 2 was enriched for both CREB dependent and independent to a similar extent $\left(p=7.9 \mathrm{e}^{-17}\right.$ and $p<1 \mathrm{e}^{-50}$ for CREB independent and CREB dependent, respectively), and cluster 3 showed enrichment of previously defined CREBdependent genes (Lakhina et al., 2015; $p=1.2 \mathrm{e}^{-24}$; Fig. 5f). Altogether, it is likely that both aversive and positive olfactory conditioning induce stimulus-independent gene expression changes related to memory, with a second group of differentially expressed genes being specific to the learning and memory assay used.

To confirm the implication of the identified genes in memory, we selected mutant worm strains for 10 genes from clusters 1 and 2 and tested their role in aversive LTAM. As a control, we first tested the impact of the deletion of the candidate genes on the chemotaxis toward diacetyl. Four of the mutants showed reduced chemotaxis, whereas the remaining six had no significant alteration in their attraction to DA (Fig. 6a). In the case of syd-2(lf), the chemotaxis to DA was severely impaired, which did not allow further analysis of the LTAM. Therefore, we tested the role of the remaining nine candidates on aversive olfactory learning and LTAM. None of the genes tested showed a defect in learning, whereas five demonstrated significant impairment of LTAM memory. Surprisingly, we found that loss of $r p m-1$ improves LTAM, which suggests that $r p m-1$ may regulate forgetting (Fig. 6b).

\section{Discussion}

In the current study, we investigated temporal gene regulation after aversive LTAM in C. elegans. We found that gene activation occurs in several waves after training. Based on the actinomycin D pulse treatment, two time points seem to be critical for establishment of long-term memory; an immediate and a second $4 \mathrm{~h}$ gene activation wave. Interestingly, most of the genes in cluster 2 that show a major activation $4 \mathrm{~h}$ after conditioning are also transiently activated immediately after conditioning, which first decreased before peaking again at $4 \mathrm{~h}$ after training. This biphasic activation pattern together with the effect of transient inhibition of transcription at 3-4 h after training with actinomycin $\mathrm{D}$, support the idea that several transcriptional activation waves regulate long-term memory. However, our results cannot fully exclude that the delayed high expression of some transcripts might be attributed to increased mRNA stability induced after conditioning. In addition, we cannot clearly define the hierarchical connections between the observed transcriptional activation events; thus, it is possible that the initial gene activations are prerequi- 
sites for later transcriptional regulation processes and sequential, interdependent transcriptional activation waves act in concert during LTAM. Interestingly, based on clustering analysis of transcriptionally activated genes, we identified a third gene activation wave peaking at $8 \mathrm{~h}$ after treatment; however, this seems dispensable for LTAM maintenance. Previously, a biphasic CREB activation pattern has been suggested in the dentate gyrus of rats and during fear conditioning in mice (Schulz et al., 1999; Trifilieff et al., 2006), suggesting multiple transcriptional activation waves. Our data are in line with these results, suggesting an evolutionary conserved multiphasic gene activation mechanism during memory formation. In addition to the temporal profiling, we also introduced a second filtering step to eliminate genes activated during aversive conditioning unrelated to memory, and we built a core gene set that is more likely memory specific. Yet, we observed that the downregulated genes are less consistent across experiments and associate mostly with metabolic GO terms and are likely independent of olfactory learning but linked to stress and metabolic changes in the animals.

We also compared the core aversive olfactory memoryregulated gene set defined here with the results of the previous study by Lakhina et al. (2015) that uses a substantially different memory task in C. elegans. Interestingly, we found a large overlap between the genes regulated in the different assays, suggesting that there is a gene set that is activated during memory formation irrespectively of the training mode. Thus, these genes may represent general memory-regulated targets, whereas other groups of genes might be specific to the memory task applied. Alternatively, some genes are activated only at a defined time window and were not detected previously.

Finally, we analyzed the role of CREB and found that aversive LTAM is CREB dependent. This is in line with previous results demonstrating the role of CREB in different long-term memories in worms (Kauffman et al., 2011; Timbers and Rankin, 2011; Lakhina et al., 2015), mollusks, flies, and vertebrates (Yin and Tully, 1996).

Although CREB is essential for memory in all species investigated so far (Frank and Greenberg, 1994), the time points at which CREB activity is required are less understood. Phosphorylation of Ser133 has been identified as the main essential regulator of CREB (Yamamoto et al., 1990) and Western blot analysis demonstrated a biphasic increase in phosphorylation, for example, in the hippocampus (Trifilieff et al., 2006). However, a recent study using Ser133 mutant mice demonstrated that phosphorylation of this serine site is not needed for memory (Briand et al., 2015). Previously, Kauffman et al. (2011) demonstrated that the C. elegans CREB homolog crh-1 is phosphorylated at the conserved serine site during positive olfactory LTAM. In accordance with vertebrate studies and previous results from C. elegans, we found that $c r h-1$ is phosphorylated during aversive LTAM. The temporal kinetics of $c r h-1$ phosphorylation coincides with the immediate gene activation peak, suggesting that CREB plays a crucial role directly after learning. The possible immediate role of CREB activity is also supported by the temporal rescue of $\mathrm{crh}-1$ mutant worms (Fig. $5 d$ ), which shows that $c r h-1$ is necessary directly after conditioning. However, our results cannot rule out the possibility that the early CREB activation is required for a later, also CREB-dependent transcriptional activation that occurs at later time points. Future studies will also be necessary to investigate whether the transcriptional activation of the genes induced by the different LTAM assays shows the same dependence on CREB. Altogether, in the current study we identified a core memory-regulated gene set that contains general condition- ing independent as well as aversive LTAM-regulated genes that are regulated, at least in part, by an evolutionary conserved early CREB-dependent gene activation mechanism in C. elegans aversive LTAM.

\section{Notes}

Supplemental material for this article is available at http://mon.unibas. ch/files/Celegans_LTAM_Expression_SuppMaterial.zip. This material has not been peer reviewed.

\section{References}

Ardiel EL, Rankin CH (2010) An elegant mind: learning and memory in Caenorhabditis elegans. Learn Mem 17:191-201. CrossRef Medline

Bargmann CI, Hartwieg E, Horvitz HR (1993) Odorant-selective genes and neurons mediate olfaction in C. elegans. Cell 74:515-527. CrossRef Medline

Benoit CE, Rowe WB, Menard C, Sarret P, Quirion R (2011) Genomic and proteomic strategies to identify novel targets potentially involved in learning and memory. Trends Pharmacol Sci 32:43-52. CrossRef Medline

Brenner S (1974) The genetics of Caenorhabditis elegans. Genetics 77:7194. Medline

Briand LA, Lee BG, Lelay J, Kaestner KH, Blendy JA (2015) Serine 133 phosphorylation is not required for hippocampal CREB-mediated transcription and behavior. Learn Mem 22:109-115. CrossRef Medline

Burger C, Lopez MC, Baker HV, Mandel RJ, Muzyczka N (2008) Genomewide analysis of aging and learning-related genes in the hippocampal dentate gyrus. Neurobiol Learn Mem 89:379-396. CrossRef Medline

Cavallaro S, D'Agata V, Manickam P, Dufour F, Alkon DL (2002) Memoryspecific temporal profiles of gene expression in the hippocampus. Proc Natl Acad Sci U S A 99:16279-16284. CrossRef Medline

Croce A, Cassata G, Disanza A, Gagliani MC, Tacchetti C, Malabarba MG, Carlier MF, Scita G, Baumeister R, Di Fiore PP (2004) A novel actin barbed-end-capping activity in EPS-8 regulates apical morphogenesis in intestinal cells of Caenorhabditis elegans. Nat Cell Biol 6:1173-1179. CrossRef Medline

Frank DA, Greenberg ME (1994) CREB: a mediator of long-term memory from mollusks to mammals. Cell 79:5-8. CrossRef Medline

Gautier L, Cope L, Bolstad BM, Irizarry RA (2004) affy-analysis of Affymetrix GeneChip data at the probe level. Bioinformatics 20:307-315. CrossRef Medline

Hastie RT, Narasimhan B, Chu G (2014) PMAR: Pam: prediction for microarrays. Available at: http://CRAN.R-project.org/package=pamr.

Huang da W, Sherman BT, Lempicki RA (2009) Systematic and integrative analysis of large gene lists using DAVID bioinformatics resources. Nat Protoc 4:44-57. CrossRef Medline

Kaletsky R, Lakhina V, Arey R, Williams A, Landis J, Ashraf J, Murphy CT (2016) The C. elegans adult neuronal IIS/FOXO transcriptome reveals adult phenotype regulators. Nature 529:92-96. CrossRef Medline

Kauffman A, Parsons L, Stein G, Wills A, Kaletsky R, Murphy C (2011) C. elegans positive butanone learning, short-term, and long-term associative memory assays. J Vis Exp pii:2490. CrossRef Medline

Lakhina V, Arey RN, Kaletsky R, Kauffman A, Stein G, Keyes W, Xu D, Murphy CT (2015) Genome-wide functional analysis of CREB/longterm memory-dependent transcription reveals distinct basal and memory gene expression programs. Neuron 85:330-345. CrossRef Medline

Langfelder P, Zhang B, Horvath S (2008) Defining clusters from a hierarchical cluster tree: the Dynamic Tree Cut package for R. Bioinformatics 24:719-720. CrossRef Medline

Paratore S, Alessi E, Coffa S, Torrisi A, Mastrobuono F, Cavallaro S (2006) Early genomics of learning and memory: a review. Genes Brain Behav 5:209-221. CrossRef Medline

Park CS, Gong R, Stuart J, Tang SJ (2006) Molecular network and chromosomal clustering of genes involved in synaptic plasticity in the hippocampus. J Biol Chem 281:30195-30211. CrossRef Medline

Pfaffl MW (2001) A new mathematical model for relative quantification in real-time RT-PCR. Nucleic Acids Res 29:e45. CrossRef Medline

Ritchie ME, Phipson B, Wu D, Hu Y, Law CW, Shi W, Smyth GK (2015) limma powers differential expression analyses for RNA-sequencing and microarray studies. Nucleic Acids Res 43:e47. CrossRef Medline

Roy PJ, Stuart JM, Lund J, Kim SK (2002) Chromosomal clustering of muscle-expressed genes in Caenorhabditis elegans. Nature 418:975-979. CrossRef Medline 
Ryan MM, Mason-Parker SE, Tate WP, Abraham WC, Williams JM (2011) Rapidly induced gene networks following induction of long-term potentiation at perforant path synapses in vivo. Hippocampus 21:541-553. CrossRef Medline

Schulz S, Siemer H, Krug M, Höllt V (1999) Direct evidence for biphasic cAMP responsive element-binding protein phosphorylation during long-term potentiation in the rat dentate gyrus in vivo. J Neurosci 19:5683-5692. Medline

Stetak A, Hörndli F, Maricq AV, van den Heuvel S, Hajnal A (2009) Neuronspecific regulation of associative learning and memory by MAGI-1 in C. elegans. PLoS One 4:e6019. CrossRef Medline

Timbers TA, Rankin CH (2011) Tap withdrawal circuit interneurons require CREB for long-term habituation in Caenorhabditis elegans. Behav Neurosci 125:560-566. CrossRef Medline

Trifilieff P, Herry C, Vanhoutte P, Caboche J, Desmedt A, Riedel G, Mons N, Micheau J (2006) Foreground contextual fear memory consolidation requires two independent phases of hippocampal ERK/CREB activation. Learn Mem 13:349-358. CrossRef Medline

Von Stetina SE, Watson JD, Fox RM, Olszewski KL, Spencer WC, Roy PJ, Miller DM 3rd (2007) Cell-specific microarray profiling experiments reveal a comprehensive picture of gene expression in the C. elegans nervous system. Genome Biol 8:R135. CrossRef Medline

Vukojevic V, Gschwind L, Vogler C, Demougin P, de Quervain DJ, Papassotiropoulos A, Stetak A (2012) A role for alpha-adducin (ADD-1) in nematode and human memory. EMBO J 31:1453-1466. CrossRef Medline

Yamamoto KK, Gonzalez GA, Menzel P, Rivier J, Montminy MR (1990) Characterization of a bipartite activator domain in transcription factor CREB. Cell 60:611-617. CrossRef Medline

Yin JC, Tully T (1996) CREB and the formation of long-term memory. Curr Opin Neurobiol 6:264-268. CrossRef Medline 